\title{
TILINGS IN TOPOLOGICAL SPACES
}

\section{F. G. ARENAS}

(Received 11 September 1996 and in revised form 6 May 1997)

\begin{abstract}
A tiling of a topological space $X$ is a covering of $X$ by sets (called tiles) which are the closures of their pairwise-disjoint interiors. Tilings of $\mathbb{R}^{2}$ have received considerable attention (see [2] for a wealth of interesting examples and results as well as an extensive bibliography). On the other hand, the study of tilings of general topological spaces is just beginning (see [1, 3, 4, 6]). We give some generalizations for topological spaces of some results known for certain classes of tilings of topological vector spaces.
\end{abstract}

Keywords and phrases. Tiling, topological space, star-finite tiling, singular points, facets, vertices.

1991 Mathematics Subject Classification. 52.

1. Introduction. A tiling of a topological space $X$ is a covering of $X$ by sets (called tiles) which are the closures of their pairwise-disjoint interiors.

Tilings of $\mathbb{R}^{2}$ have received considerable attention (see [2] for a wealth of interesting examples and results as well as an extensive bibliography). On the other hand, the study of tilings of general topological spaces is just beginning (see [1, 3, 4, 6]).

By way of notation, for a subspace $A$ of a topological space $X$, the symbols $\bar{A}, \operatorname{Fr} A$, and $A^{\circ}$ denote, respectively, the closure, boundary, and interior of $A$. For a collection $\mathscr{A}$ of subsets of a set $X$, we denote by, $\cup \mathscr{A}$ and $\cap \mathscr{A}$, respectively, the union and intersection of the members of $\mathscr{A}$. For a point $x \in X$, we define $\mathscr{A}(x)=\{A \in \mathscr{A}: x \in A\}$.

The following definitions are basic to our discussion. Let $\mathscr{T}$ be a tiling of a topological space $X$. We define the set of frontier points of $\mathscr{T}$ to be the union of the boundaries of the tiles in $\mathcal{T}$ and denote this set by $F(\mathscr{T})$. The protected points of $\mathscr{T}$ constitute the set $P(\mathscr{T})=\left\{x \in X: x \in(\bigcup \mathscr{T}(x))^{\circ}\right\}$ and the complement of this set in $X$ is $U(\mathscr{T})$, the set of unprotected points of $\mathcal{T}$. The set of improper points of $\mathcal{T}$ is defined as $I(\mathscr{T})=\{x \in X: \mathscr{T}(x)=\{T\}$ with $x \in \operatorname{Fr} T\}$. The set of 2-protected points is $P(\mathscr{T})_{2}$, the subset of protected points common to exactly two tiles.

A singular point of $\mathscr{T}$ is a (frontier) point at which $\mathscr{T}$ is not locally finite (that is, a point every neighborhood of which intersects infinitely many tiles in $\mathscr{T})$. The collection of all singular points of $\mathscr{T}$ is denoted by $S(\mathscr{T})$. Finally, we define $S_{0}(\mathscr{T})=\{x \in S(\mathscr{T})$ : $\mathscr{T}(x)$ is infinite .

The relations between the sets defined above have been established in [4, 6] (for topological vector spaces, but they remain valid for topological spaces) and are summarized in the following proposition.

Proposition 1.1. For any tiling $\mathcal{T}$ of any topological space $X$, the following inclusions hold: $S(\mathscr{T}) \cap P(\mathscr{T}) \subset S_{0}(\mathscr{T}) \subset S(\mathscr{T}) \subset F(\mathscr{T})$ and $I(\mathscr{T}) \subset U(\mathscr{T}) \subset S(\mathscr{T})$. Moreover, 
$F(\mathscr{T})$ and $S(\mathscr{T})$ are closed sets.

None of these inclusions can be improved, as [4, Ex. 1.1] shows.

2. Star-finite tilings. A tiling is called star-finite if each tile meets only finitely many other tiles.

Two tilings $\mathscr{T}$ and $\mathscr{T}_{1}$ of the same space $X$ are said to be topologically equivalent if and only if there exists a homeomorphism $h: X \rightarrow X$ such that if $T \in \mathcal{T}$, then $h(T) \in \mathcal{T}_{1}$ (thus, also if $T_{1} \in \mathscr{T}_{1}$, then $h^{-1}\left(T_{1}\right) \in \mathscr{T}$ ).

Nielsen defined in [4] four properties that a tiling in a vector topological space could satisfy. Only two of these properties have sense in topological spaces and are quoted below.

A tiling $\mathcal{T}$ of a topological space $X$ satisfies property $\mathbf{P}_{3}$ if and only if for each $T \in \mathscr{T}$, $x \in \operatorname{Fr} T$, and neighborhood $U$ of $x$, there is an open neighborhood $V$ of $x, V \subset U$, such that $V-\operatorname{Fr} T$ has exactly two connected components.

A tiling $\mathscr{T}$ of a topological space $X$ satisfies property $\mathbf{P}_{4}$ if and only if for each proper subcollection $\mathscr{S} \subset \mathcal{T}$ and each pair of distinct tiles $T_{1}$ and $T_{2}$ in $\mathscr{Y}$, the set $T_{1} \cap T_{2} \cap \operatorname{Fr}(\bigcup \mathscr{S})$ is nowhere dense in $\operatorname{Fr}(\bigcup \mathscr{Y})$.

It is proved in [4] that $\mathbf{P}_{3}$ implies $\mathbf{P}_{4}$ and one can easily see that the proof given there for topological vector spaces remains valid for arbitrary topological spaces. The reverse implication is false.

We denote, by Cond $A$, the set of condensation points of $A$. The following result is the first part of [4, Thm. 3.1].

THEOREM 2.1. If $\mathscr{T}$ is a star-finite tiling of a topological space, then $U(\mathscr{T})=S(\mathscr{T}) \supset$ $\overline{S_{1}(\mathscr{T})} \supset \operatorname{Cond}\left(S_{1}(\mathscr{T})\right)$.

The following lemma is quoted from [4, Lem. 3.3].

LEMMA 2.2. Suppose $M$ and $N$ are closed subsets of a topological space such that $M=\operatorname{Cond}(M), N \subset M$, and $N$ is nowhere dense in $M$. Then $M=\operatorname{Cond}(M-N)$.

THEOREM 2.3. If $\mathcal{T}$ is a star-finite tiling that possesses $\mathbf{P}_{3}$ of a topological space such that no connected open set can be disconnected by deleting a countable subset, then $U(\mathscr{T})=S(\mathscr{T})=\overline{I(\mathscr{T})}=\operatorname{Cond}(I(\mathscr{T}))$.

Proof. The proof is essentially the same as that of [4, Thm. 3.4]. We only have to note that the role of the point 0 in that proof can be equally replaced by any other point of $X$ and that we can avoid the reference to [4, Lem. 3.2] (which uses essentially the vector structure) using the additional hypothesis over $X$ and over $\mathcal{T}$ (in [4, Thm. 3.4] it is only supposed that $\mathscr{T}$ is $\mathbf{P}_{4}$ ) in the following way.

We need to show that $M \subset$ Cond $M$, where $M$ is the frontier of the union of a finite set of tiles. If this were not true, then there would be a point $x \in M$ such that every open neighborhood of it intersects $M$ in a countable set of points. Let $T$ be a tile such that $x \in \operatorname{Fr} T$. By $\mathbf{P}_{3}$, we have that if $V$ is a connected open neighborhood of $x$, $V-\operatorname{Fr} T=V-(V \cap \operatorname{Fr} T)$ has two components, contradicting both the fact that the set $V \cap \operatorname{Fr} T$ is countable and the hypothesis over $X$.

Note that the topological hypothesis imposed on $X$ is verified by every topological 
vector space of dimension greater than one. And the more restrictive hypothesis of being $\mathcal{T} \mathbf{P}_{3}$ instead of $\mathbf{P}_{4}$ is easier to check than the other.

3. Tilings and density of certain classes of frontier points. We can easily note that [6, Thm. 1.4, Cor. 1.5, Thm. 1.6, Cor. 1.7] do not need the vector structure. Hence, they can be stated as follows.

THEOREM 3.1. Let $X$ be a topological space such that every closed subset of it is a Baire space in the subspace topology (for example, completely metrizable spaces). Let $\mathcal{T}$ be a countable tiling of $X$ with property $\mathbf{P}_{3}$. Then the set $P_{2}(\mathscr{T}) \cap R(\mathscr{T})$ is dense and open in $F(\mathcal{T})$.

COROLLARY 3.2. If $X$ and $\mathcal{T}$ are as in Theorem 3.1, the set $S(\mathcal{T})$ of singular points of $\mathcal{T}$ is nowhere dense in $F(\mathcal{T})$.

THEOREM 3.3. If $X$ and $\mathscr{T}$ are as in Theorem 3.1 and $\mathscr{T}$ is a countable tiling of $X$ with property $\mathbf{P}_{4}$ and with $F(\mathscr{T})-I(\mathscr{T})$ of second category in $F(\mathscr{T})$ at each point of $F(\mathscr{T})$, then $P_{2}(\mathscr{T})$ is dense and open in $F(\mathscr{T})$.

COROLLARY 3.4. If $X$ and $\mathcal{T}$ are as in Theorem 3.3, the set $S(\mathcal{T})$ of singular points of $\mathcal{T}$ (and thus $I(\mathscr{T})$ ) is nowhere dense in $F(\mathscr{T})$.

Note that Corollaries 3.2 and 3.4 have the same thesis for two noncomparable hypotheses $\left(\mathbf{P}_{3}\right.$ implies $\mathbf{P}_{4}$, but it does not imply that $F(\mathscr{T})-I(\mathscr{T})$ is of second category in $F(\mathscr{T})$ at each point of $F(\mathscr{T})$ ).

On the other hand, Nielsen [6, Thm. 1.8] has a topological thesis, but the vector structure is essential in the hypothesis.

THEOREM 3.5. Let $X$ be a separable normed space such that every closed subspace of it is a Baire space in the subset topology. Let $\mathcal{T}$ be a countable tiling of $X$ such that any line that sections $\mathcal{T}$ contains at most countably many points of $F(\mathcal{T})$. Then $P_{2}(\mathcal{T})$ is dense and (relatively) open in $F(\mathscr{T})$.

A carefull study of the proof shows that the vector hypothesis is used once in order to establish a lemma with topological thesis and supposed that thesis, the rest of the proof is valid for topological spaces. The lemma needs the following definition.

DEFINITION 3.6. Let $X$ be a topological space. A tiling $\mathcal{T}$ on $X$ is called connected if given an open set $V$ intersecting $F(\mathscr{T})$, the set of points of $V$ common to exactly two tiles in $\mathscr{T}$ is of second category in $V \cap F(\mathscr{T})$.

And, hence, Theorem 2.3.12 of Nielsen's Ph.D. Thesis states the following:

LEMMA 3.7. Let $X$ be a separable normed space such that every closed subspace of it is a Baire space in the subset topology. Let $\mathcal{T}$ be a countable tiling of $X$ such that any line that sections $\mathcal{T}$ contains at most countably many points of $F(\mathcal{T})$. Then the tiling is connected.

For topological spaces, we have the following results:

LEMмA 3.8. Let $X$ be a topological Baire space, $\mathcal{T}$ a connected countable tiling, and let $U$ be an open set in $X$ intersecting $F(\mathscr{T})$. Then there are two tiles $T_{1}$ and $T_{2}$ in $\mathcal{T}$ and 
an open set $V \subset U$ intersecting $F(\mathscr{T})$ such that $V \cap F(\mathscr{T}) \subset T_{1} \cap T_{2}$ and such that the set $\left\{x \in V: \mathscr{T}(x)=\left\{T_{1}, T_{2}\right\}\right\}$ is dense in $V \cap F(\mathscr{T})$.

Proof. The proof is the same as in Nielsen's Ph.D. Thesis for the case of normed separable spaces, but as it does not appear in [3, 4], or [6], we quote it here for the sake of completeness.

From the hypothesis, the set of points of $V$ common to exactly two tiles in $\mathscr{T}$ is of second category in $U \cap F(\mathscr{T})$. Since $\mathscr{T}$ is countable and $F(\mathscr{T})$ is closed (and, thus, is a Baire space by assumption), it follows, from the Baire category theorem, that for some two tiles $T_{1}$ and $T_{2}$ in $\mathcal{T}$, the set of points common to exactly $T_{1}$ and $T_{2}$ is not nowhere dense in $U \cap F(\mathscr{T})$. Thus, there is an open set $V \subset U$ intersecting $F(\mathscr{T})$ such that the points in $V$ common to exactly the tiles $T_{1}$ and $T_{2}$ are dense in $V \cap F(\mathscr{T})$. But $T_{1} \cap T_{2}$ is closed, so we must also have $T_{1} \cap T_{2} \supset V \cap F(\mathscr{T})$.

THEOREM 3.9. Let $X$ be a topological Baire space and $\mathcal{T}$ a connected countable tiling. Then $P_{2}(\mathscr{T})$ is dense and (relatively) open in $F(\mathscr{T})$.

Proof. Again, the proof is the same as in Nielsen's Ph.D. Thesis.

That $P_{2}(\mathscr{T})$ is relatively open in $F(\mathscr{T})$ follows immediately from the definitions. Let $U$ be a connected open set in $X$ intersecting $F(\mathscr{T})$. We show that $U$ contains points of $P_{2}(\mathscr{T})$, which proves the theorem.

By the preceding lemma, there are two tiles $T_{1}$ and $T_{2}$ in $\mathscr{T}$ and an open set $V \subset U$ intersecting $F(\mathscr{T})$ such that $V \cap F(\mathscr{T}) \subset T_{1} \cap T_{2}$ and such that the set $E_{T_{1}, T_{2}}=\{x \in$ $\left.V \cap F(\mathscr{T}): \mathscr{T}(x)=\left\{T_{1}, T_{2}\right\}\right\}$ is dense in $V \cap F(\mathscr{T})$. Let $T^{i}=T_{1} \cup T_{2}$ and define a new tiling $\mathscr{T}^{i}$ of $X$ by $\mathscr{T}^{i}=\left\{T^{i}\right\} \cup\left(\mathscr{T}-\left\{T_{1}, T_{2}\right\}\right)$.

Assume, to reach a contradiction, that $V \cap F\left(\mathscr{T}^{i}\right)=V \cap F(\mathscr{T})(\neq \varnothing)$.

Applying again the preceding lemma, we obtain a tile $T_{3} \in \mathscr{T}^{i}-\left\{T^{i}\right\}=\left(\mathscr{T}-\left\{T_{1}, T_{2}\right\}\right)$ and an open set $W \subset V \subset U$ intersecting $F(\mathscr{T})$ such that $W \cap F(\mathscr{T})=W \cap F\left(\mathscr{T}^{i}\right) \subset T_{3}$.

This, however, contradicts the fact that $E_{T_{1}, T_{2}}$ is dense in $U \cap F(\mathscr{T})$. Thus, we must have $V \cap\left(F(\mathscr{T})-F\left(\mathscr{T}^{i}\right)\right) \neq \varnothing$. Since $F(\mathscr{T})-F\left(\mathscr{T}^{i}\right) \subset P_{2}(\mathscr{T})$, the proof is now complete.

COROLLARY 3.10. Let $X$ be a topological Baire space and $\mathcal{T}$ a connected countable tiling. Then $S(\mathscr{T})$ is nowhere dense in $F(\mathscr{T})$.

Compare with Corollaries 3.2 and 3.4.

4. Facets and vertices of a tiling. To complete our study of tilings of topological spaces, we are going to consider a generalization of a result by Breen that Nielsen did not consider in the realm of topological vector spaces.

Given a tiling $\mathscr{T}$ of a topological space $X$, a facet of the tiling is a connected component of the intersection of some finite set of tiles. We say that a facet is degenerated if it is a singleton (in that case, we call that facet a vertex). In any other case, we say that the facet is nondegenerated. (In the case of countable tilings by topological disc, these definitions correspond to those of [1].)

Call $D(\mathscr{T})$ the set of points in $F(\mathscr{T})$ that are not in a nondegenerated facet of $\mathscr{T}$. In [1], one can find a relation between the cardinals of $D(\mathscr{T})$ and $S(\mathscr{T})$ for countable tilings of the plane by closed topological discs. 
We show that one of the two parts of that relation can be extended to topological spaces with weak restrictions on the tiling and that the other is false even for countable tilings by topological balls of the euclidean space.

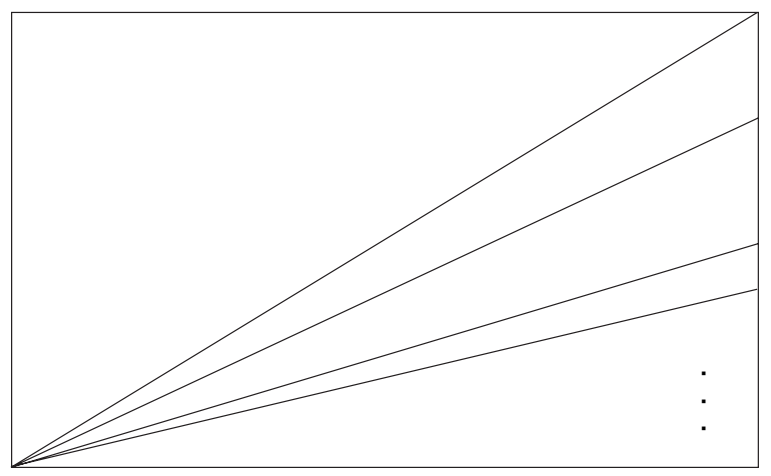

FiguRE 1. A tiling in $\mathbb{R}$.

THEOREM 4.1. Let $X$ be a topological space and $\mathcal{T}$ a tiling such that $\operatorname{Fr} T$ is locally connected for each tile $T \in \mathcal{T}$ and suppose that for every $T \in \mathcal{T}$, every $x \in \operatorname{Fr} T$ and every neighborhood $U$ of $x$, the set $(U-\{x\}) \cap \operatorname{Fr} T$ is nonvoid. Then $D(\mathscr{T}) \subset S(\mathscr{T})$, and so, $|D(\mathscr{T})| \leq|S(\mathscr{T})|$.

Proof. Let $x$ be a point of $D(\mathscr{T})$. We show that $x$ is a singular point. Since $I(\mathscr{T}) \subset$ $S(\mathscr{T})$ by Proposition 1.1, it is enough to show that $x$ is an improper point.

In case every neighborhood of $x$ contains a boundary point of $\mathcal{T}$ which belongs only to a tile $T$, one can use the above case to construct a net of singular points converging to $x$, which completes the proof.

Now, given a point $x \in D(\mathscr{T})$, we can assume that $x \in \operatorname{Fr} T$ for certain $T \in \mathscr{T}$ and given any neighborhood $U$ of $x$, every point of $\operatorname{Fr} T \cap U$ belongs to at least two tiles.

Choose $x_{1} \in \operatorname{Fr} T \cap(U-\{x\})$ (since the hypothesis ensures that the set is nonvoid) and a tile $T_{1} \neq T$ with $x_{1} \in T_{1}$. Suppose that there is some neighborhood $V$ of $x$ such that Fr $T \cap V \subset T \cap T_{1}$. By the hypothesis of local connectedness, we can suppose that Fr $T \cap V$ is connected, and so, it is contained in a connected component of $T \cap T_{1}$. Thus, Fr $T \cap U$ is not a singleton, which means that $x$ belongs to a nondegenerate facet and so $x \notin D(\mathscr{T})$. So for every neighborhood $U$ of $x$, there is some point $x_{2}$ of $\operatorname{Fr} T \cap V-\{x\}$ with $x_{2} \notin T_{1}$. Take again the neighborhood $U$ and select a tile $T_{2} \neq T_{1}, T$ with $x_{2} \in T_{2}$. An induction yields prove that $U$ has points in infinitely many different tiles. Hence, $x$ is a singular point.

This result is clearly an extension of [1, Lem. 1, Cor. 1]. One can ask if the other part of the theorem ([1, Lems. 2 and 3, Cor. 2]) can be extended to topological spaces under suitable hypothesis. The answer is completely negative since we can construct in the following example a countable tiling of $\mathbb{R}^{3}$ by topological balls that does not satisfy [1, Cor. 2]. 
EXAMPLE 4.2. Take the product with $[0,1]$ of the tiling by triangles given in Figure 1 and extend to a countable tiling of $\mathbb{R}^{3}$ adding cubes. All the points of the product with $[0,1]$ of the set of singular points of that tiling are singular in the tiling of $\mathbb{R}^{3}$, but all of them are in a nondegenerated facet.

ACKnowledgement. The author is indebted to Professor Dr. Mark J. Nielsen, who kindly provided $[3,4,6]$ and his Ph.D. Thesis. The author is partially supported by DGICYT grant PB95-0737 and Plan Propio de Investigacion de la Universidad de Almería.

\section{REFERENCES}

[1] M. Breen, A characterization theorem for tilings having countably many singular points, J. Geom. 21 (1983), no. 2, 131-137. MR 85f:52033. Zbl 539.52014.

[2] B. Grunbaum and G. C. Shephard, Tilings and Patterns, W. H. Freeman and Company, New York, 1987. MR 88k:52018. Zbl 601.05001.

[3] M. J. Nielsen, Singular points of a convex tiling, Math. Ann. 284 (1989), no. 4, 601-616. MR 90g:52016. Zbl 662.52008.

[4] _ Singular points of a star-finite tiling, Geom. Dedicata 33 (1990), no. 1, 99-109. MR 91b:52015. Zbl 703.52009.

[5] _ Tilings of topological vector spaces, Ph.D. thesis, University of Washington, 1990.

[6] __ On two questions concerning tilings, Israel J. Math. 81 (1993), no. 1-2, 129-143. MR 94i:52020. Zbl 780.52001.

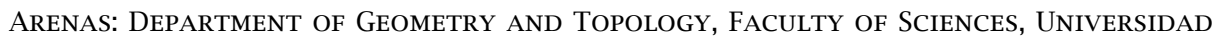
DE ALMERÍA, 04071 ALMERÍA, SPAIN 


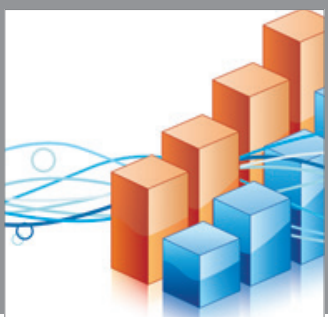

Advances in

Operations Research

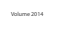

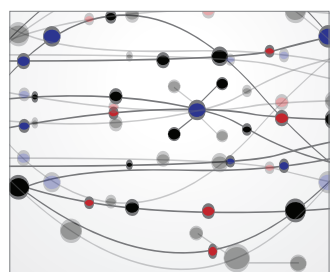

\section{The Scientific} World Journal
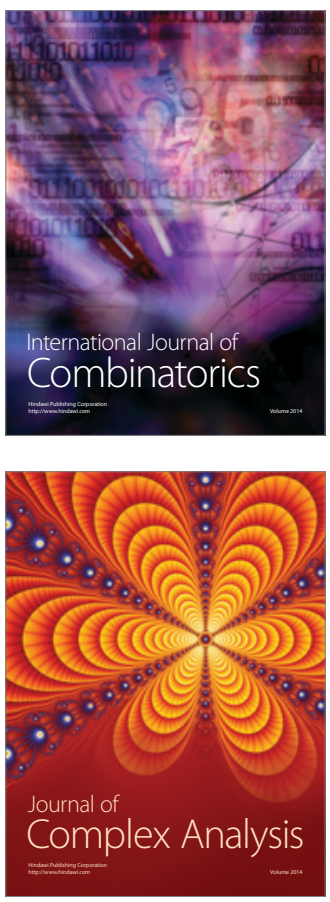

International Journal of

Mathematics and

Mathematical

Sciences
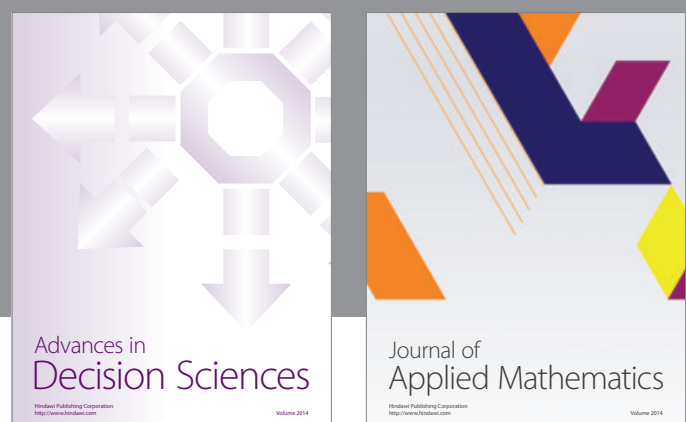

Journal of

Applied Mathematics
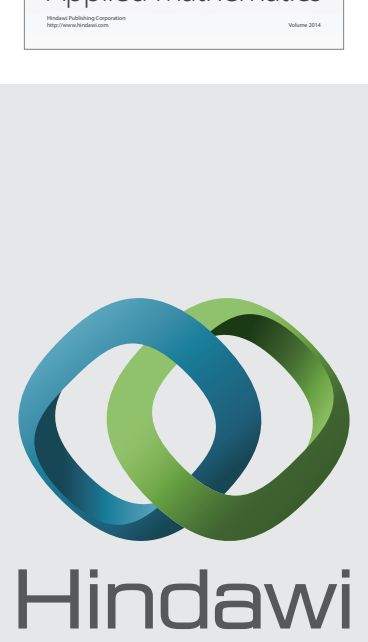

Submit your manuscripts at http://www.hindawi.com
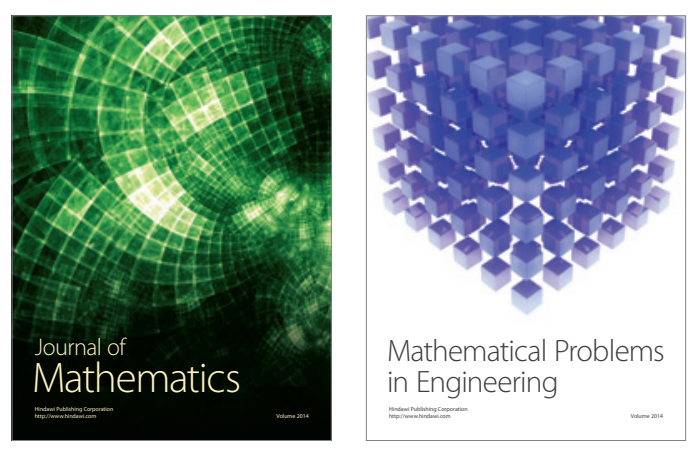

Mathematical Problems in Engineering
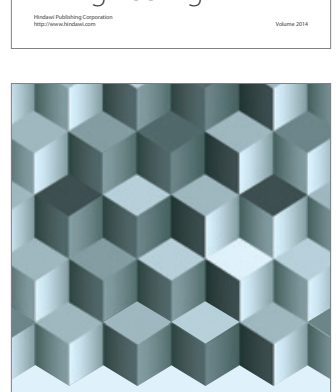

Journal of

Function Spaces
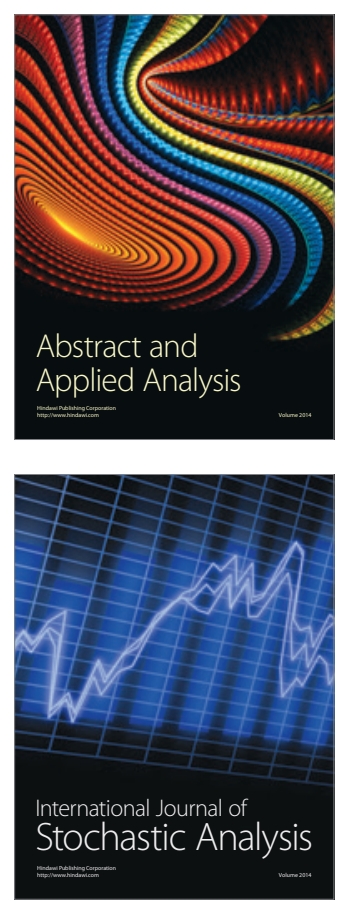

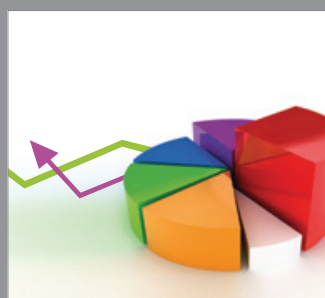

ournal of

Probability and Statistics

Promensencen
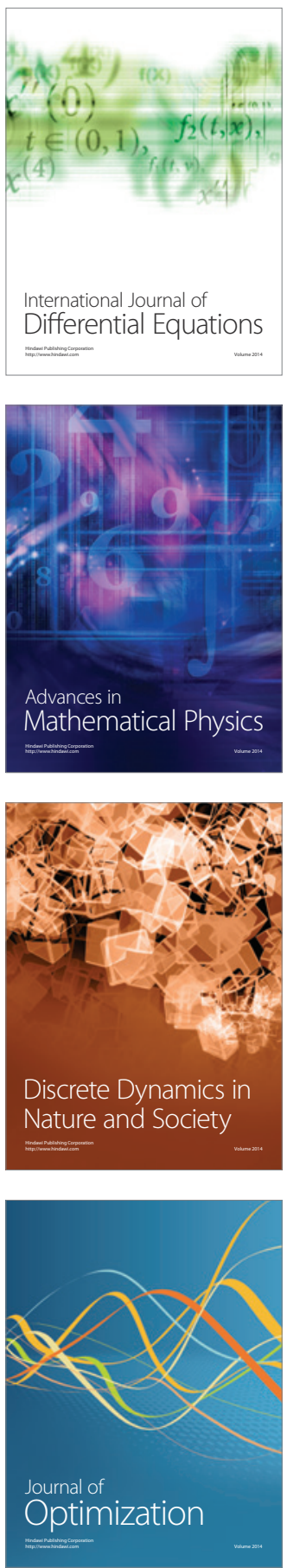